\title{
Defects Identification and Assessment in Engineering Education Using Lean Tools
}

\author{
Zainab Al-Baldawi ${ }^{1}$, Dhuha Kadhim Ismayyir ${ }^{2}$ \\ 1,2 Department of Production and Metallurgy Engineering / University of Technology, Baghdad - Iraq
}

\begin{abstract}
Today, lean philosophy is widely used in many manufacturing and service systems to reduce or eliminate waste and defects for improving system quality. Recently, Lean Engineering Education (LEE) is a new concept lean philosophy and widely used in education system. Engineering education is so important field where practical and theoretical study have a strong impact to increase students' knowledge and improve their skills. This study will illustrate how can be successfully used Lean tools on the engineering education system by identifying defects that have a negative influence on the final quality of the theoretical and practical studies. E- questionnaire by google form is prepared to collect data about quality of engineering education elements from students point view where it was conducted in mechanical engineering department and included 82 students. Six engineering education elements that have a strong impact on the quality of the theoretical and practical studies are used, namely; lecturer, students, curriculum, assessment process, scientific method and scientific equipment and tools. Lean tools, namely; pareto analysis, fishbone analysis have been used to clarify the defects types and causes of the critical defect in the engineering education that have a big influence on the quality of both studies. weights method is used also to compare between the theoretical and practical studies to identify which one is less quality than another quality scale was used that based on triple Likert scale to identify the quality level; namely bad, acceptable and good. The results showed that both the theoretical and practical study in the mechanical engineering department have the same acceptable level of quality and the scientific equipment and tools have largest defect in the practical study and the unavailable enough number of laboratory devices is considered a critical cause of scientific equipment and tool defects but in the theoretical study, the assessment process has largest defects with critical causes result from unclear the assessment questions during examinations.
\end{abstract}

Keywords: Engineering education, Fishbone diagram, Pareto analysis, Practical Study, Theoretical Study, Weights method.

\section{Introduction}

Engineering education is so important aspect in engineering that focused on both the theoretical and practical studies to deliver information, knowledge and improvement the skills of students. Engineering Education can be defined

"A systematic, student-centred and valueenhanced approach to educational service delivery that enables students to holistically meet, lead and shape industrial, individual and societal needs by integrating comprehension, appreciation and application of tools and concepts of engineering fundamentals and professional practice" [1].

The engineering faculties continuously work to improve education quality is defined as "the ability of student's knowledge to satisfy stated requirements [2]. Many quality improvement techniques can be applied for improving performance of the engineering education. 
One of the possible ways for improving the engineering education is lean concept where lean is defined as "a dynamic process of change driven by a systematic set of principles and best practices aimed at continuous improvement. Although Lean tools differ from application to application but always the goal is quality and performance improvement where Lean focuses on eliminating or reducing anything that not satisfy the final customer [1].

The lean engineering education LEE is a modern concept that appeared recently where it uses the same principles of lean through elimination or reduction waste or defects in the engineering education systems where, defect can be defined as any activity that have negative influence on the final quality of the system. [3]. Defects in engineering education may be defined any elements of the education process that fail to perform its function or perform with bad quality.

The engineering education elements are similar than the elements in the manufacturing process where; equipment and tools $=$ machine, curriculum $=$ raw material, lecturer = worker, assessment process $=$ measurement, scientific method= method. Some Engineering education systems are considered the students as customers, other system saw students as raw material or even as employees. Students as raw material are going through the process to become a final product and Students like customers where they purchase the service of education. In addition, students as employees are those that should be involved in their studies and they should be motivated to perform effectively and those students are evaluated at the end. So, the quality of student's performance should be important for a university in the same way in which the quality of an employee's performance is important for a company [2].

Many researchers have studied the lean in various aspects of the engineering education;

K.G. Durga Prasad and et.al. [2.] used six sigma five phase methodology DMAIC (Define - Measure Analyze - Improve - Control) in an engineering educational institution in addition to use process capability indices, Fish bone diagram, Pareto diagram and Failure mode effect analysis to improve quality. Daniela Pusca and et.al. [4]. explained how lean principles can be used to improve an engineering design course in terms of methods of instruction, content, and assessment methods where Lean tools are used like; value stream mapping, root cause analysis and Kaizen were to understand the problems. Dragan Pavlović and et.al. [5] applied the Lean Six Sigma method on the educational process at the Faculty of Mechanical Engineering, University of Niš by defining negative defects that have impact on the quality evaluation(exam) and how these defects can be remedied. Pareto analysis, the Statistical Process Control and Ishikawa diagram are used. Suhas Ambekar [6] illustrated the areas of applying lean philosophy to improve delivery of learning experience into management education. Sylvie Nadeau [7] illustrated that in review paper how universities response to adopt a new approach of lean six sigma for managing their activities. Meddaoui Anwar and et al. [8] proposed a new manufacturing educational model that based on return of experiments and the previous works by comparing between two type of teaching.

In the engineering education, there is gap still exists between theoretical and practical studies. A gap that can be partially filled through a study the defects that existence in both to improve and generate integration between them. The cause of a failed knowledge transfer and skill improvement in engineering Education is often the shortage in the practical application. The acquisition of competence goes beyond the purely theoretical knowledge. It also includes the ability to apply knowledge to solve practical problems. Only when the connection is guaranteed between knowledge and ability for its application, we can speak of competence [9].

This research was focused on using lean tools in the theoretical and practical study in mechanical engineering department through identify the defects in the six principles engineering education elements namely lecturer, students, curriculum, assessment process, scientific method and equipment and tools. Lean tools are used namely, fishbone and pareto analysis both studies. Weights method is used to evaluate and assess of quality and performance of both studies.

\section{Methodology of the Research}

An electronic questionnaire was designed and conducted and included 82 mechanical engineering students form the second, third and fourth stages. The questionnaire included two parts. The first part of the questionnaire was carried to evaluate the quality influence of the six engineering education elements namely; lecturer, student, assessment method, curriculum, scientific method and equipment and tools using the triple Likert scale namely; strong effect, medium effect, and weak effect and the students asked to express their opinion on the quality of the six engineering education elements ,then the data was analyzed to weight the strength and importance of each element using weighted mean. The second part of the questionnaire was conducted using 
Likert Triple Likert Scale namely; agree, neutral and disagree to express student's opinion about the quality of each component of each element for the practical and theoretical study for the purpose of identifying defects after that data analysis and mean weight for each element and component is calculated. The element weight that calculated in the first questionnaire is used in weights method in addition to the weighted mean of each element for practical and theoretical studies is used also in this method to evaluate and assess which study is less quality than another. A quality scale that uses three level bad, acceptable and good quality was adapted using triple Likert scale so, results of the weights method is positioned in the adapted quality scale for each result to identify quality level for theoretical and practical study. Fishbone diagrams were used to illustrate the defects that have a strong influence in the six elements one for practical study and one for the theoretical study. Pareto analysis also was used to represented the types of defects according to quantity of defects(disagree) according to student opinion in questionnaire and also used to identify the critical cause of the highest defect in both studies. the methodology of the research was showed in Figure 1.

\section{Results and Discussion}

Data of the questionnaire for two parts were analyzed as follows: -

1- Part one of the questionnaire: the weighted mean for each element and its components were calculated according to student point view using triple Likert scale namely; agree, neutral and disagree to identify the position of each element according to this scale, where disagree range is $1-1.66$, neutral range is $1.67-2.33$ and agree rang is 2034-3 as illustrate in table 1.

\begin{tabular}{lcc}
\hline \multicolumn{1}{c}{$\begin{array}{c}\text { Engineering } \\
\text { education element }\end{array}$} & $\begin{array}{c}\text { Weighted Mean } \\
\text { of the theoretical } \\
\text { Study }\end{array}$ & $\begin{array}{c}\text { Weighted Mean } \\
\text { of Practical } \\
\text { Study }\end{array}$ \\
\hline Lecturer & 2.32 & 2.348 \\
Student & 2.516 & 2.386 \\
Curriculum & 2.008 & 1.808 \\
Assessment Process & 1.765 & 2.095 \\
Scientific Method & 2.094 & 2.415 \\
$\begin{array}{l}\text { Equipment and } \\
\text { Tools }\end{array}$ & 1.871 & 1.549 \\
\end{tabular}

Table 1 and figure 2 showed that for the theoretical study, the higher weighted mean is 2.516 for student and leatest weighted mean for assessment process equal 1.765 However, the practical sturdy, the scientific method has highest weighted mean equals 2.415 where the leatest weighted mean is 1.549 for equipment and tools.

2- The second part of the questionnaire: The student were asked their opinion to assess the quality of each element of the engineering education using triple Likert scale strong effect, medium effect and weak effect for calculating the element weight, Figure 3 shows the result of this part of the questionnaire where weighted mean, variance, standard deviation and the element weight were calculated.

Table 1 Weighted mean for most related elements theoretical and practical Studies 


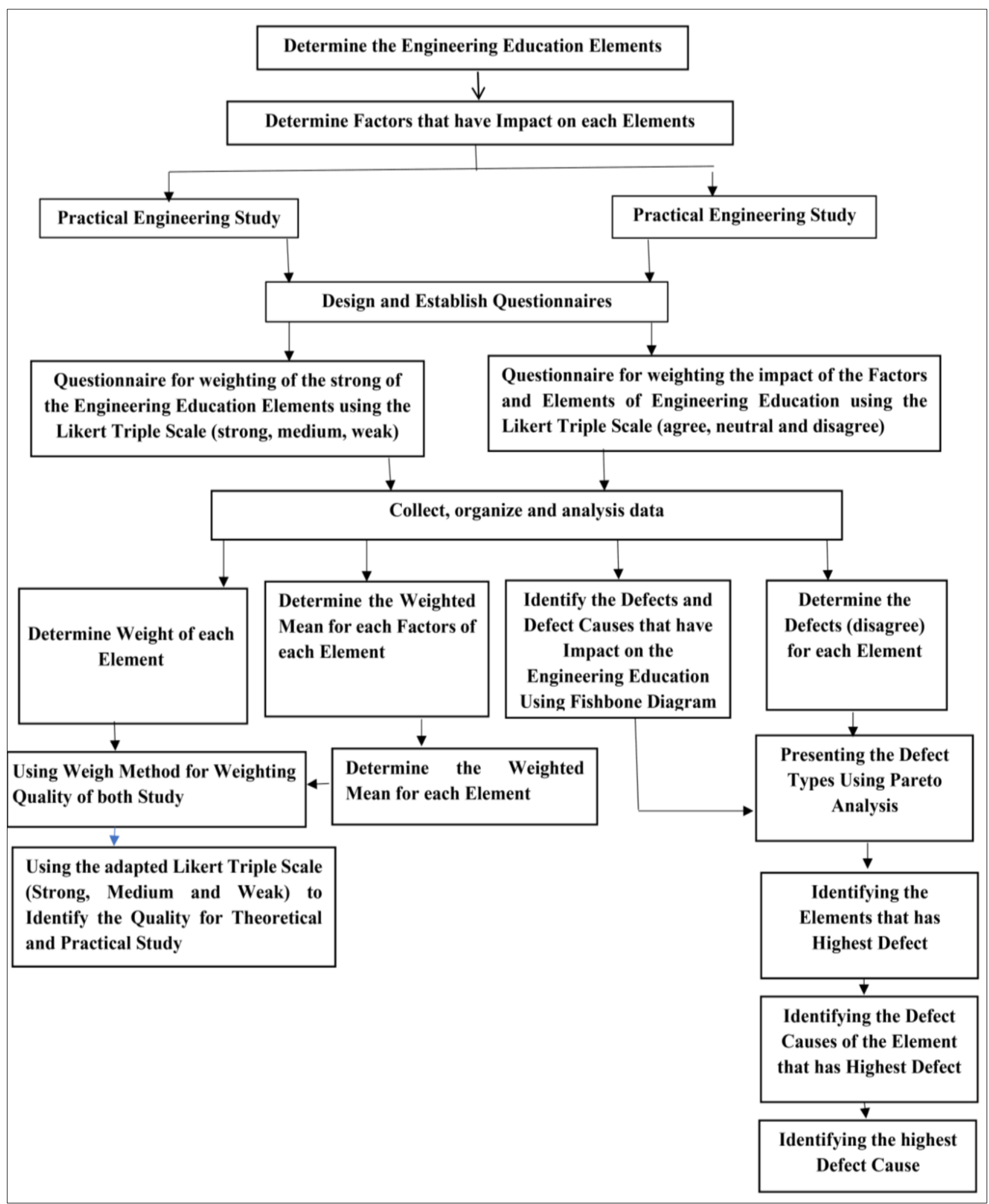

Fig. 1. Method of the Research 
Fig. 2 Comparison the Weighted Mean for both Studies

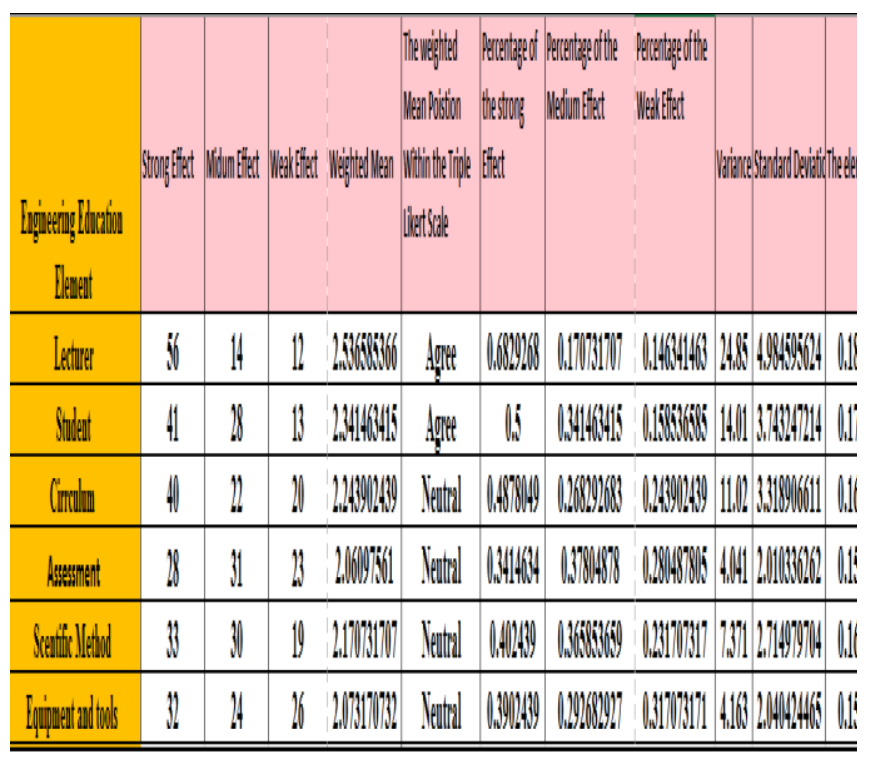

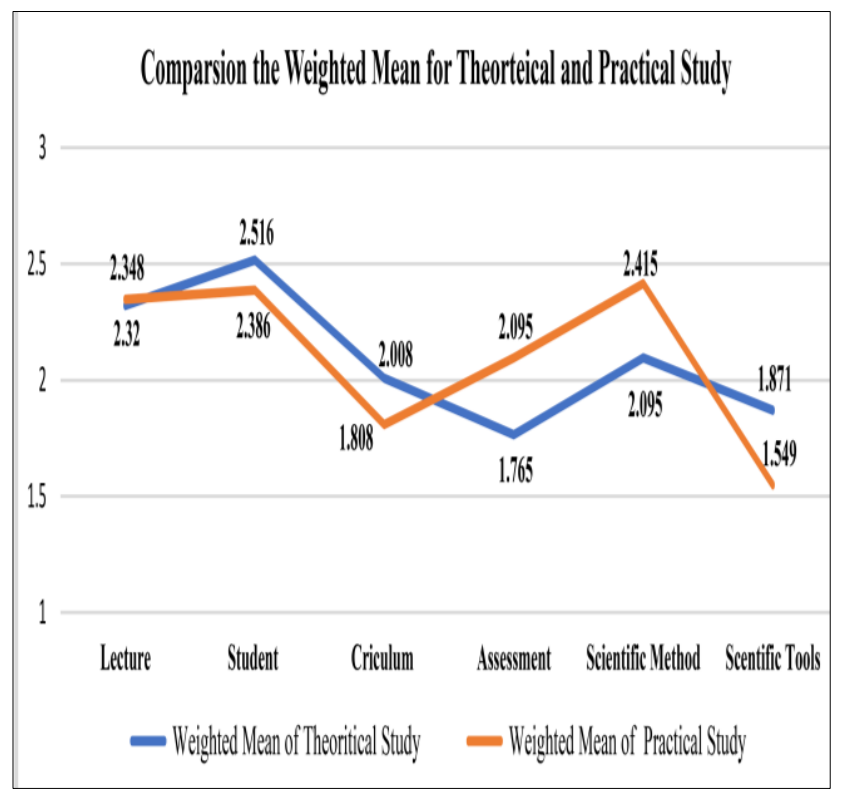

Fig. 3 Results of the Second Part of the Questionnaire.

Defects of elements components are represented it by fishbone diagram for the practical study to clarify the essential defects and its causes for the six engineering education elements as illustrated in figure 4 in the same way, the defects of the theoretical study were identified by fishbone diagram to illustrate the main defects and its causes for the six engineering education elements components for the theoretical study as shown in figure 5. 


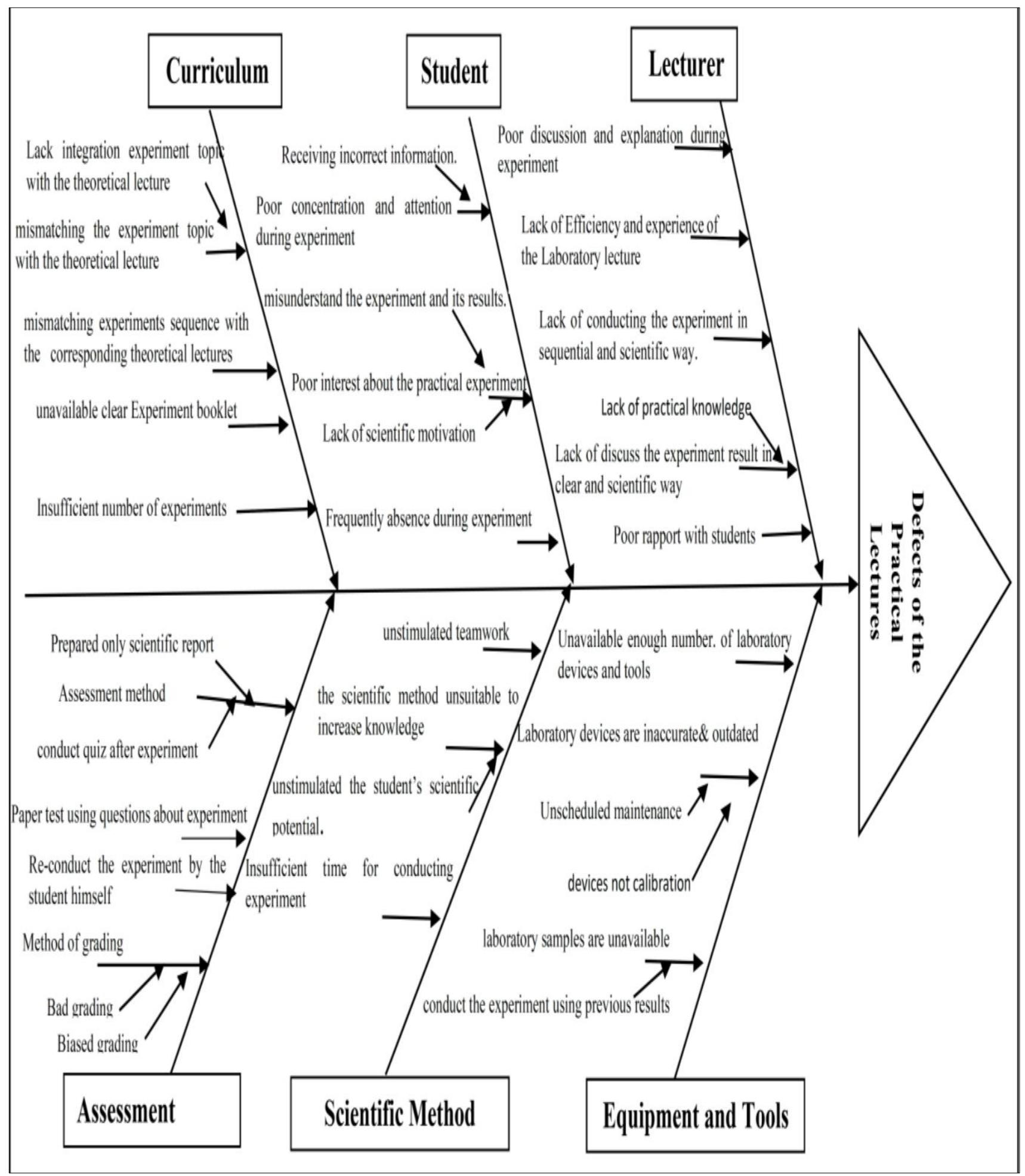

Fig. 4 Possible Causes of Defects in the Practical Lectures 


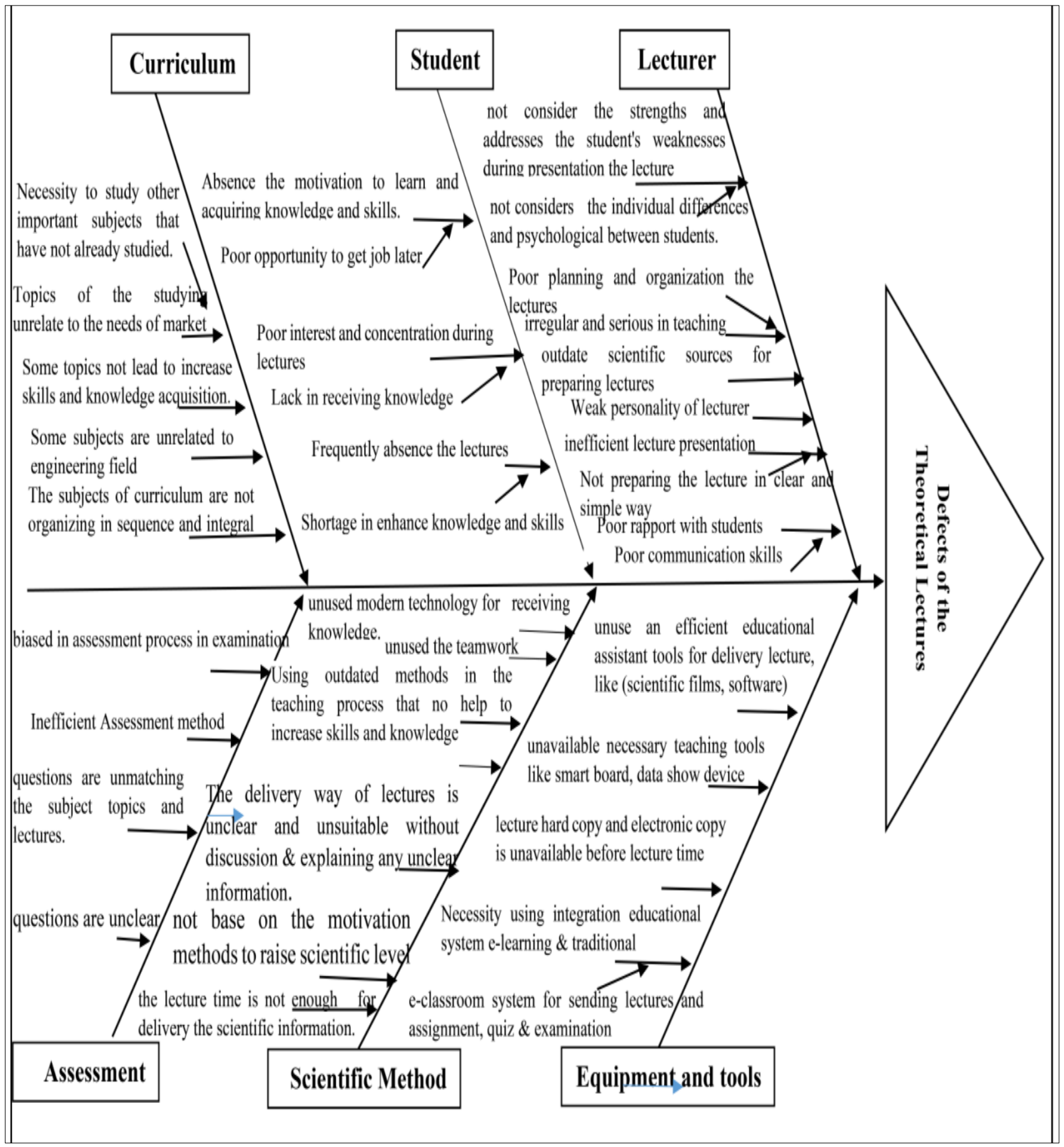

Fig.5 Possible Causes of Defects for Theoretical Studies 
Weight's method was used as a tool for comparison the quality level of the theoretical and practical studies using elements weights and weighted mean for each element of theoretical and practical studies as illustrated in Figure 6. Figure 7 represent the elements weights and show the differences between the element's weights for both studies. The results show that the theoretical study has quality $=2.1111$ and the practical study has quality= 2.1130 and both nearly have the same value of quality.

\begin{tabular}{|c|c|c|c|c|c|}
\hline Crethis & Weigght & Theoritical & Thertical Weight & Practical & Practical INeight \\
\hline Lecture & 0.18892 & 2.32 & 0.43892908 & 2,348 & 0.443581812 \\
\hline Student & 0.1739 & 2516 & 0.438757692 & 2386 & 0.416087382 \\
\hline Crichlum & 0.16112 & 2008 & 0.335778968 & 1.808 & 0,302157768 \\
\hline Assessment & 0.1335 & 1.765 & 0.170922105 & 2.095 & 0.3215156215 \\
\hline Scienfific IIethod & 0.16616 & 2005 & 0.338700745 & 2.415 & 0,390435365 \\
\hline Scentific Tools & $0.154+1$ & 1.871 & 0.288891735 & 1.549 & 0.239173345 \\
\hline Sum & 1 & & 2.111143445 & & 2.113008987 \\
\hline
\end{tabular}

Fig. 6 Comparison both Studies using Weight Method.

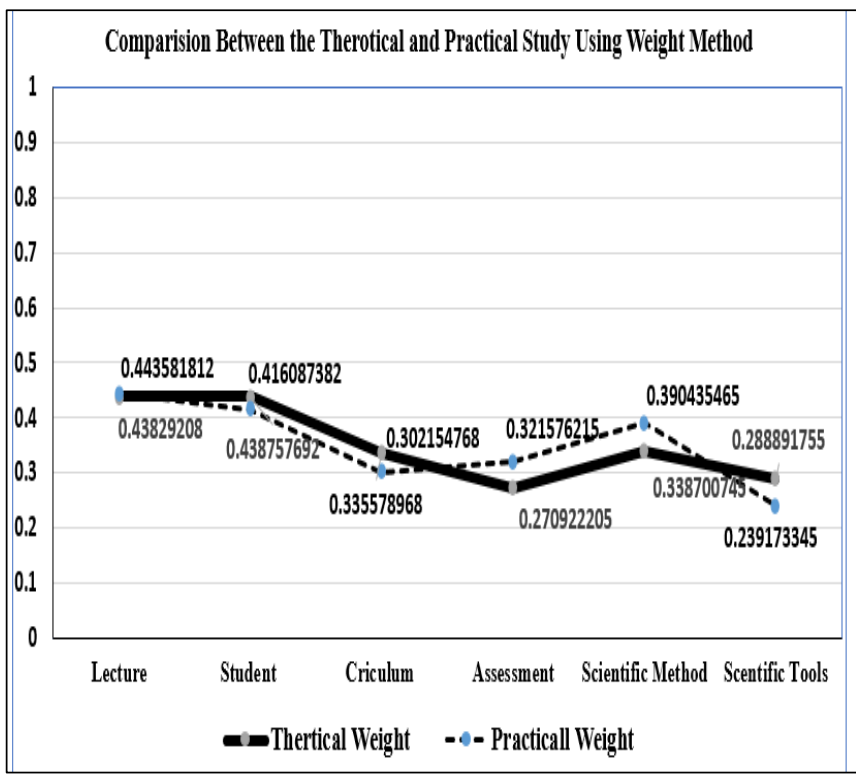

Fig. 7 Comparison between both Studies Using Weight Method.

A quality scale was adopted based on the triple Likert scale, where (1-1.66) is corresponded to bad quality, (1.67-2.33) is equal to acceptable quality and (2.34-3) is represented to good quality. Where the value of the quality for the theoretical and practical studies was represented on this scale as shown in the figure 8 . The quality level of the two studies is almost equal and is at acceptable quality level. Therefore, to improve the quality of the two studies, the quality of the elements of engineering education should be improved together with a work to reduce or eliminate the defects currently present in the elements of engineering education as shown in the fish diagram for the two studies and start improving the quality with the most critical elements in practical study and theoretical study.

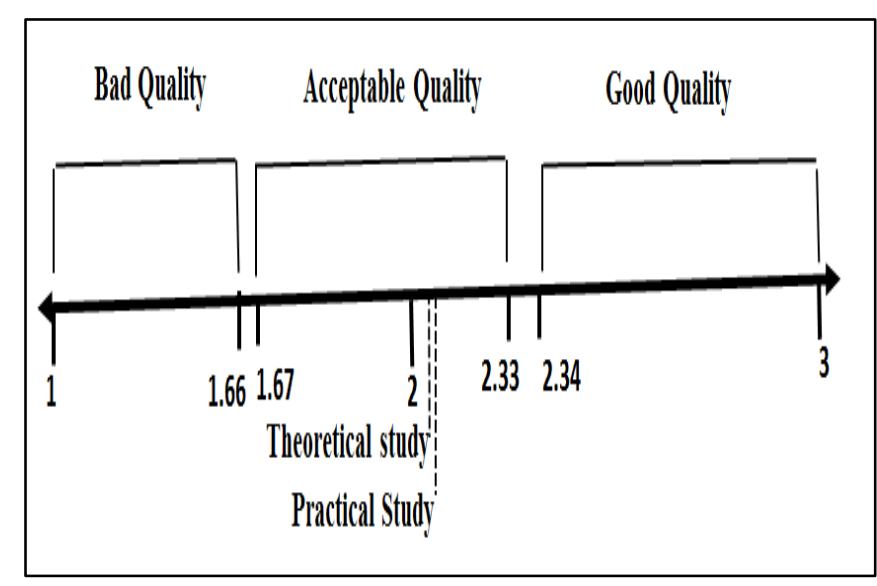

Fig. 8 Quality Level Using Adapted Triple Likert Scale.

Pareto analysis was used to present defects in each element, where disagree according Likert scale was considered as a defect, figure 9. illustrates the quantity of disagree that the student considered in each element in the practical study. Element Equipment and tools have highest defects than other elements $=165$ defect. In other side, the assessment process element has highest defects in the theoretical study than other elements = 168 defects, as shown in figure 10.

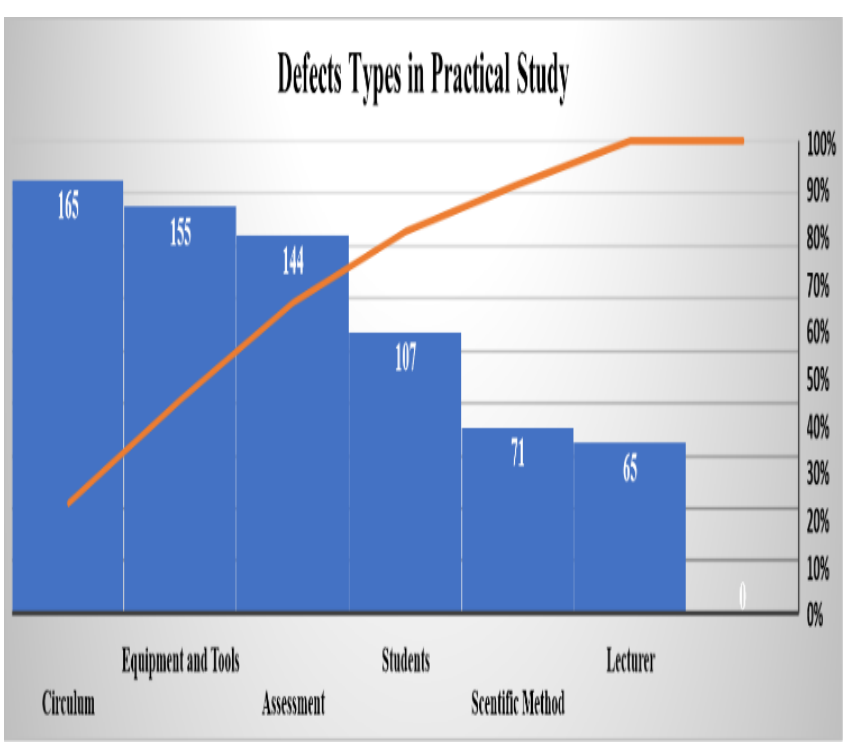

Fig. 9 Defect Types in the Practical Study 


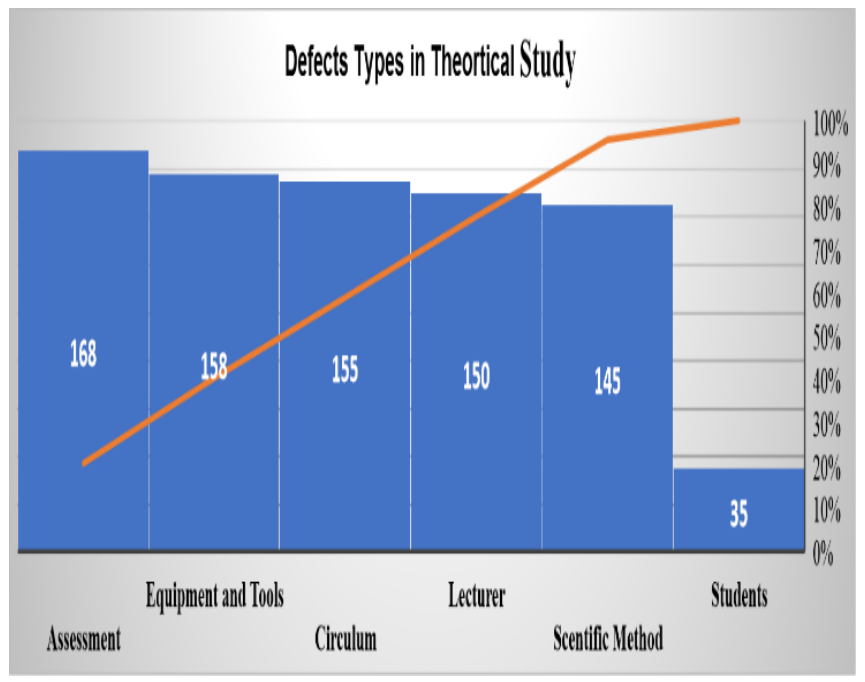

Fig. 10 Defect Types in the Theoretical Study

The causes of the highest defect in the practical study namely are unavailable enough number of laboratory devices, laboratory devices are inaccurate and outdated and unavailable laboratory samples to conduct experiments, so represent the data related to causes of Equipment and tools show that the highest cause is unavailable enough number of laboratory devices $=60$ as shown in figure 11 .

In the theoretical study, the highest cause of the assessment process defect is equations of examination for assessment student $=54$ as illustrate in figure 12 .

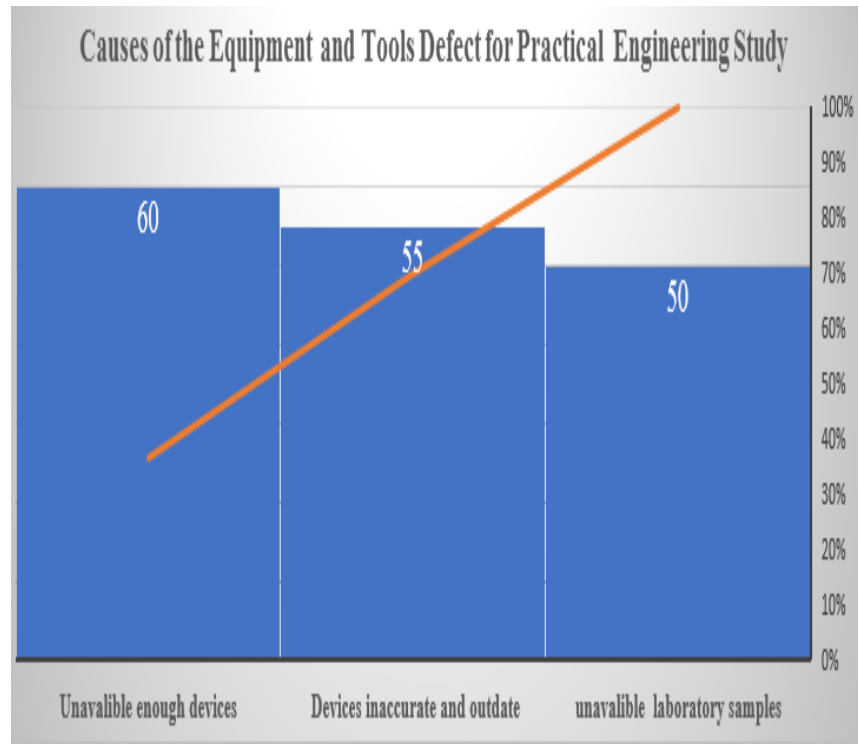

Fig. 11 Causes of Equipment and Tools Defect for Practical Study

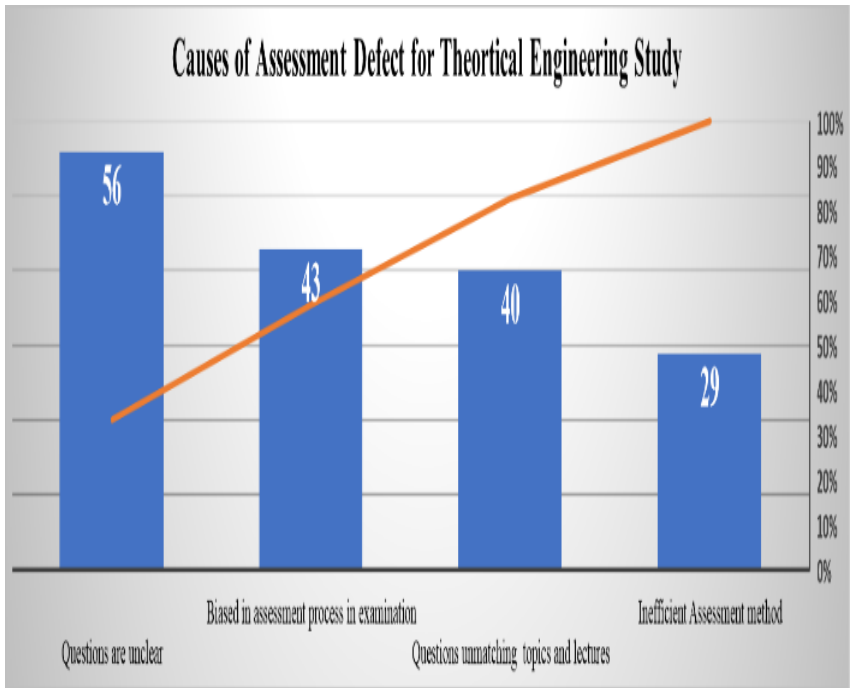

Fig. 12 Causes of Assessment Defects for Theoretical Study

\section{Conclusions}

This research may provide the basic framework in other educational field at any university for other assessment studies to analyze and improve any defects in it. Many important points can be concluded from this research: -

1. Necessity to reduce or eliminate any defect or waste in any element of the engineering education using lean tools.

2. Many defects have been detected in elements of the engineering education both in the theoretical and practical studies using fishbone diagram.

3. Weights method showed that both the theoretical and practical studies have nearly the same quality or work with same performance.

4. Many causes led to the different types of defects in the six elements in both theoretical and practical studies.

5. The critical cause of the highest defect in the six elements that has negative impact on performance of the specific element was identified using Pareto analysis to review and take corrective action to improve it. Questions unclear in the assessment examination is the critical cause in the theoretical study and unavailable enough laboratory devices is the biggest cause in the practical study.

6. Integration between theoretical and practical studied is so important to ensure delivery knowledge and skill in a good way to students.

7. the quality scale that depends on Likert scale was used to identify the quality of performance of both 
studies. The quality level of both studies is acceptable.

\section{References}

A. C. Alves; F. J. Kahlen; S. Flumerfelt and . S. A.B. Manalang.( 2014). The lean production multidisciplinary: from operations to education," in 7th International Conference on Production Research America, Lima, Peru.

2. K. . D. Prasad; K. V. Subbaiah and G. .Padmavathi. (2012).Application of six sigma Methodology in an engineering educational Institution. Int. J. Emerg. Sci, vol. 2, no. 2, pp. 222-237.

3. N. Slack; S. Chambers and R. J. Johnston.(2016). Operation management,eighth edition, Edinburgh: Pearson educational limited.

4. Daniela Pusca and Derek O. Northwood.(2016). Can lean principles be applied to course design in engineering education. Global Journal of Engineering Education, vol. 18, p. 3.

5. Dragan Pavlović; Milena Todorović, Srđan Mladenović and Peđa Milosavljević.(2014). The role of quality methods in improving education process -case study. Serbian Journal of Management, vol. 9 , no. 2, pp. 219 - 230.

6. S. Ambekar.(2016). Exploring the application of lean principles to management education in india. International Journal of Multidisciplinary Management Studies, vol. 6, no. 6, pp. 1-13.

7. S. Nadeau.(2017). Lean, six sigma and Lean six sigma in higher education:a reveiw of experiences around the world," American Journal of Industrial and Business Management, vol. 7, pp. 591-603.

8. Meddaoui Anwar; Hachmoud Sami and Allali Hakim.(2018). Teaching Lean Manufacturing in Educational Field Through LBD: A Case Study in an Engineering School. International Journal of Vocational Education and Training Research, vol. 4, no. 1, pp. 41-44.

9. E. R. Dominik and T. Mattab.(2014). Mini-factory a learning factory concept for students and small and medium size enterprises," in Variety Management in Manufacturing. Proceedings of the 47th CIRP Conference on Manufacturing System. 